\title{
FWM-based Amplitude Limiter Realizing Phase Preservation through Cancellation of SPM Distortions
}

\author{
Kyle R. H. Bottrill ${ }^{(1)}$, Francesca Parmigiani ${ }^{(1)}$, David J. Richardson ${ }^{(1)}$, and Periklis Petropoulos ${ }^{(1)}$ \\ (1) Optoelectronics Research Centre, University of Southampton, Southampton, SO17 1BJ, UK. \\ E-mail address: K.Bottrill@soton.ac.uk
}

\begin{abstract}
We present a FWM-based amplitude regenerator that includes an optical phase predistortion stage to compensate for the effects of Kerr-induced amplitude-to-phase noise conversion of the signal. Successful operation of the scheme is demonstrated on a QPSK signal. OCIS codes: (070.5040) Phase conjugation ; (060.4370) Nonlinear optics, fibers; (200.6015) Signal regeneration.
\end{abstract}

\section{Introduction}

Phase preserving amplitude regenerators for complex modulation formats have been investigated for their potential to avoid amplitude-to-phase noise conversion due to fiber nonlinearity during transmission [1]. They can also form a useful complement to phase squeezing regenerators, many of which transfer phase noise onto the amplitude [2]. Phase preserving amplitude regeneration for phase-shift keying (PSK) signals can be achieved using either cross-phase modulation in a nonlinear amplifying loop mirror [3] or gain saturation of a pump degenerate four-wave mixing (FWM) process [4-6] in a nonlinear medium. Although amplitude regeneration through FWM saturation is not intrinsically phase preserving due to self-phase modulation (SPM) induced amplitude-to-phase noise conversion in the nonlinear medium, several operating regimes have been shown capable of minimizing this conversion [5, 6]. For example, we have recently shown that FWM saturation is favorably achieved at a high pump-to-signal power contrast ratio, since this allows the signal power to be kept at extremely low levels at the input of the HNLF [6]. In this regime, the main source of nonlinear phase noise comes from cross-phase modulation due to the presence of a strong pump [7] and if this has a high optical-signal-to-noise ratio (OSNR) and a narrow linewidth (as is typically the case), then even this noise contribution can be neglected. However, it is not always practical to operate in this regime, since it requires careful management of stimulated Brillouin scattering (SBS) effects in the HNLF prompted by the high pump power requirement [7].

In this paper, we propose and demonstrate a new scheme for phase preserving FWM-based amplitude saturation, which uses a second nonlinear stage with the purpose of compensating for the parasitic, yet deterministic effects of SPM. This reduces the demand for high pump power, in turn relaxing the HNLF requirements. Our results show effective amplitude noise cancellation in a 10 GBaud quadrature phase-shift keying (QPSK) signal, even for as high input magnitude errors as $20.2 \% \mathrm{rms}$, while inducing negligible additional phase noise penalty.

\section{Operation Principle and Experimental setup}

Saturation of FWM is an effective means of limiting the amplitude of a signal. However, unless care has been taken to avoid it, the signal undergoes SPM while propagating in the nonlinear medium in which FWM takes place. This leads directly to conversion of the amplitude noise of the signal into phase noise with deleterious effects on the quality of PSK modulation (see Fig.1 (a)). To combat this noise conversion, we propose a scheme consisting of two simple pump-degenerate FWM stages. The first stage is a wavelength converter, the role of which is to produce a phaseconjugated idler and pre-distort its phase through the onset of SPM on the signal. The phase of the idler can then be approximated (neglecting pump noise and phase) by: $\phi_{I}{ }^{\prime}=-\phi_{1}+\gamma_{I} S_{I} L_{l}$, where $\phi_{1}$ is the original signal phase, $\gamma_{l}$ is the nonlinear coefficient of the HNLF, $S_{l}$ is the signal power and $L_{l}$ is the HNLF length. The role of the second FWM stage (Fig.1 (b)) is then fourfold: 1) conversion of the conjugated copy back to the initial wavelength; 2) regeneration of the signal's amplitude through FWM saturation; 3) restoration of the signal to its original unconjugated state; 4) net cancellation of SPM-induced phase distortions that have incurred in the two stages. This can be achieved by noting that the phase of the newly generated idler at the output of the second stage is: $\phi_{2}{ }^{\prime}=$ - $\phi_{1}{ }^{\prime}+\gamma_{2} S_{2} L_{2}=\phi_{1}+\left(-\gamma_{1} S_{1} L_{1}+\gamma_{2} S_{2} L_{2}\right)$, where $\gamma_{2}$ and $L_{2}$ are respectively the nonlinear coefficient and length of the secondstage HNLF, and $S_{2}$ is the signal power at the input of the second stage. This relation shows that any phase noise caused by the onset of SPM can be cancelled out by ensuring that $\gamma_{1} S_{1} L_{I}=\gamma_{2} S_{2} L_{2}$ (in analogy, for example, to nonlinearity compensation through optical phase conjugation in the middle of a transmission link [8]). The inclusion of the two nonlinear stages has the added benefit that pump phase dithering used to effectively increase the SBS threshold in the HNLFs can be counter-balanced, so that it does not affect the final signal performance.

Our experimental set-up is shown in Fig.2, with the various stages color-coded for clarity. Firstly, light from a laser 

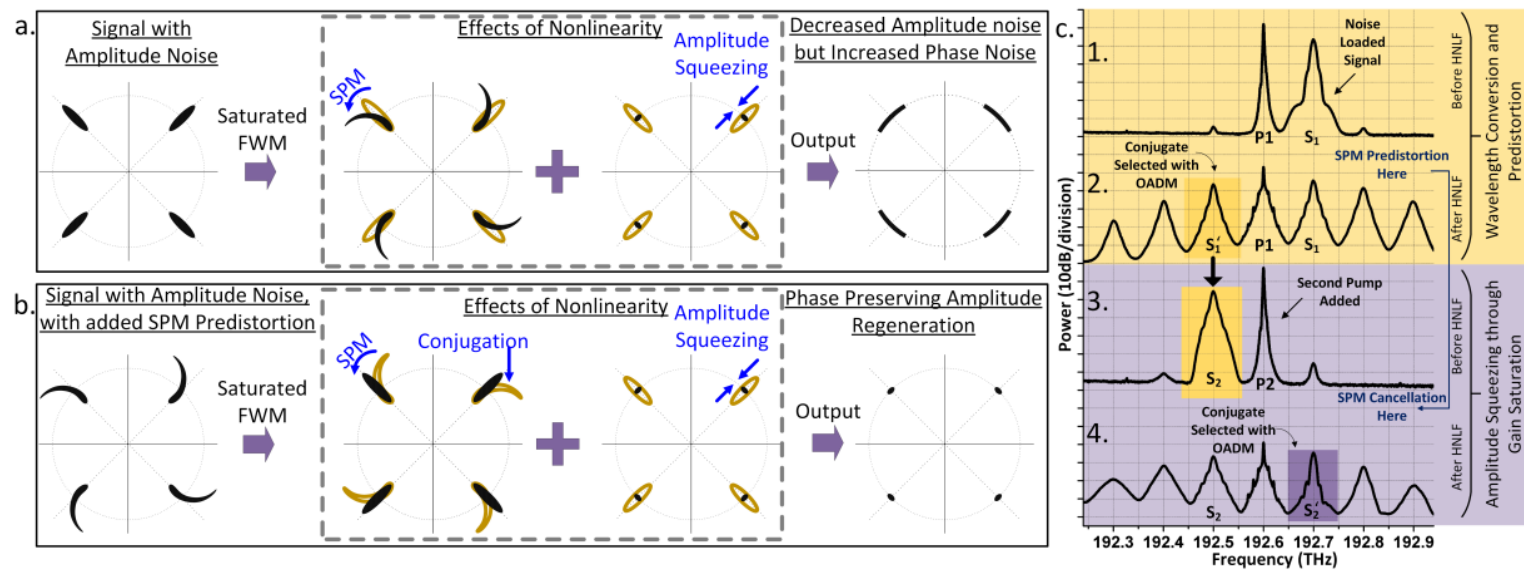

Fig. 1: Operation principle of (a) the typical and (b) proposed FWM-based phase preserving amplitude limiter. (c) 1. - 4. Examples of measured spectra at different points in the system.

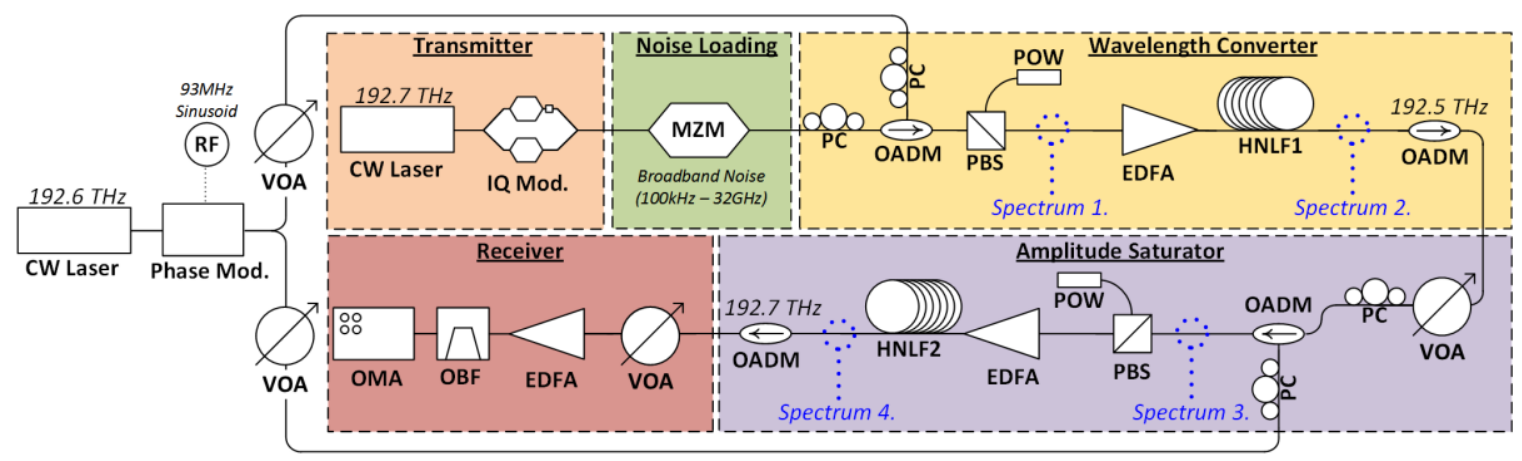

MZM=Mach-Zehnder Modulator, PC=Polarization Controller, PBS=Polarization Beam Splitter, POW=Power Meter, AWG=Arrayed Waveguide Grating, HNLF=Highly Nonlinear Fiber, OMA=Optical Modulation Analyzer, OBF=Optical Bandpass Filter, RF=Radio Frequency Signal, OADM=Optical Add-Drop multiplexer

Fig. 2 Experimental setup of the two stage FWM-based phase preserving amplitude limiter.

operating at $192.6 \mathrm{THz}$ and phase-dithered using a $93 \mathrm{MHz}$ sine wave (to increase the SBS threshold in the two HNLFs), was divided into two paths and used as the pumps (P1 and P2) in the two nonlinear stages. A second laser operating at $192.7 \mathrm{THz}$ was launched into an I-Q modulator to generate a 10 GBaud QPSK signal. The modulated signal then entered a noise-loading stage that used photo-detected broadband ASE noise to drive an amplitude modulator. It was then fed to the wavelength converting stage, and combined with P1 (see corresponding spectrum in Fig.1 (c.1)). A polarization beam splitter was used to ensure that signal and pump were co-polarized. HNLF1 comprised multiple segments of low dispersion HNLFs with a total length of $1 \mathrm{~km}$. A pump-to-signal power ratio of $1.5 \mathrm{~dB}$ was used at the input of HNLF1, with a total power of $20.5 \mathrm{dBm}$, tuned to provide the appropriate amount of SPM predistortion. The wavelength-converted phase conjugate (see Fig.1. (c.2)) was selected using an optical adddrop multiplexer (OADM) and entered the second nonlinear stage. It was there combined with the second occurrence of the pump (P2) and launched into HNLF2 for amplitude saturation and subsequent wavelength conversion (see Fig.1. (c.3) for the corresponding spectrum). As before, HNLF2 comprised multiple fiber segments of a total length of $580 \mathrm{~m}$. The total power into the fiber was $25 \mathrm{dBm}$ and the pump-to-signal peak-power ratio was $6 \mathrm{~dB}$. The output of this stage was at the original wavelength (192.7 THz - see Fig.1. (c.4)), amplitude-limited and unconjugated relative to the original. In addition, through the combined action of the two nonlinear stages, the effects of both the SPM-induced phase noise and the pump phase dither were cancelled out. The signal was selected using an OADM and analyzed using an optical modulation analyzer (OMA). The signal quality after dual stage regeneration was compared to the performance before regeneration as well as to the signal when a single stage limiter was considered by simply bypassing the wavelength converter in Fig. 2 and using the same operating power.

\section{Experimental results and Discussion}

Figure 3 (a) shows example constellation diagrams of the signal before regeneration, after single-stage regeneration and after dual-stage regeneration (the proposed scheme), for the cases when no noise and two severe cases of amplitude noise were added to the signal. The plots on the left column (no added noise) show that the amplitude 

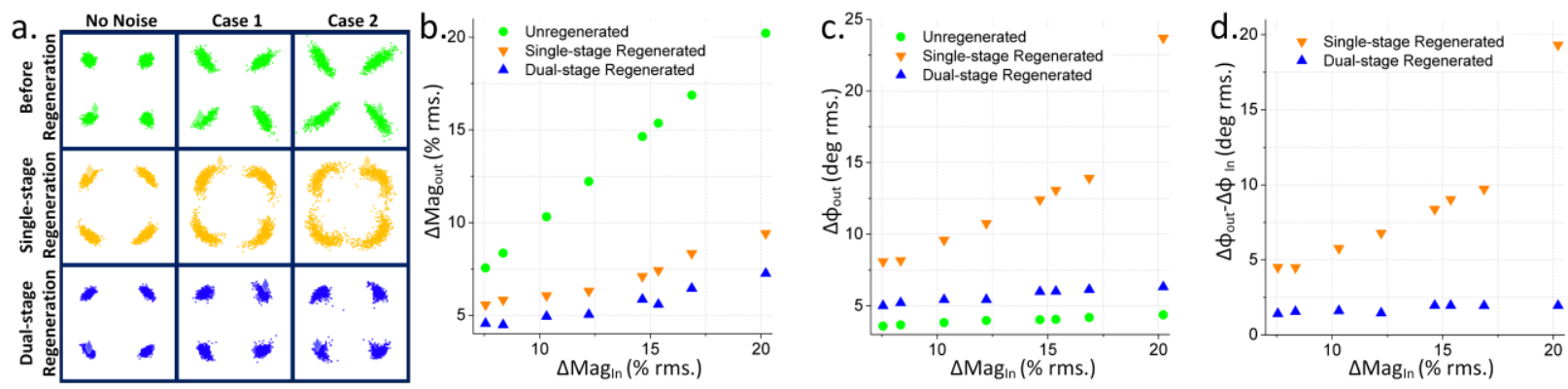

Fig. 3 Constellation diagrams (a) and noise statistics (b-d) before and after the regenerator for single and dual nonlinear stage.

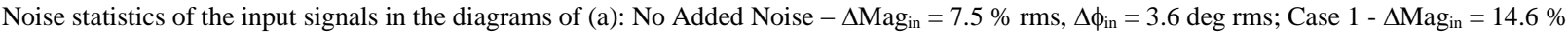
$\mathrm{rms}, \Delta \phi_{\text {in }}=4.0 \mathrm{deg} \mathrm{rms}$; Case $2-\Delta \mathrm{Mag}_{\text {in }}=20.2 \% \mathrm{rms}, \Delta \phi_{\text {in }}=4.4 \mathrm{deg} \mathrm{rms}$.

appears slightly regenerated (due to a non-ideal transmitter) in both the single stage and our dual-stage system. Phase noise on the signal however, is increased from $\Delta \phi=3.6 \mathrm{deg}$. rms to $\Delta \phi=5.0 \mathrm{deg}$. rms in the dual stage and already as a high value as $\Delta \phi=8.1 \mathrm{deg}$. rms in the single stage system. As amplitude noise at the input increases (Cases 1, 2 in Fig. 3 (a)), the amplitude noise appears drastically reduced at the output of both types of regenerators (see Fig.3 (b) for further noise cases). The effect of SPM-induced amplitude-to-phase noise conversion can be clearly seen in the results taken from the single-stage regenerator (see Fig.3 (a), Cases 1,2), where the phase noise at the output increases in accordance to amplitude noise at the input (see also Fig.3 (c, d) for further noise cases). In contrast, the phase noise at the output of the dual-stage regenerator is almost independent of the amplitude noise at the input. Figure 3 (d) suggests that the phase noise penalty suffered at the output of this stage is constant and rather small ( 1.9 deg. rms). This penalty can be attributed to OSNR degradation in the two successive nonlinear stages, induced noise from the pump, as well as imperfect cancellation of the pump dither. Finally bit-error ratio (BER) measurements on the dual-stage regenerator (not presented here) showed a $0.5 \mathrm{~dB}$ sensitivity improvement (for $\mathrm{BER}=10^{-4}$ ) for Case 1 of Fig. 3 (a) relative to the signal before regeneration, and a $0.55 \mathrm{~dB}$ improvement (for $\mathrm{BER}=10^{-4}$ ) for Case 2 . We note however, that the BER is not a suitable metric in this case, since PSK signals experience the deleterious effects of amplitude noise only after further transmission [1].

\section{Conclusions}

We have proposed and experimentally demonstrated a phase preserving FWM-based amplitude limiter using two simple pump-degenerate FWM stages. The first one is used as an optical phase pre-distortion stage to undo any SPMinduced amplitude to phase noise conversion accrued in the actual amplitude saturation stage. This drastically reduces the output phase noise, while decreasing the needed pump powers. An almost threefold reduction of the magnitude noise is demonstrated on a QPSK signal for an initial magnitude error of $20.2 \% \mathrm{rms}$, while the corresponding phase noise is maintained fairly constant as the amplitude noise increases.

\section{Acknowledgement}

The work is part of the EPSRC Photonics Hyperhighway Programme Grant. F. Parmigiani is a Royal Academy of Engineering/EPSRC Research Fellow. Open access data for this work is available at doi:10.5258/SOTON/382891

\section{References}

[1] J. P. Gordon and L. F. Mollenauer, "Phase noise in photonic communications systems using linear amplifiers", Opt. Lett. 15, 1351-1353 (1990).

[2] J. Kakande, et al., "Multilevel quantization of optical phase in a novel coherent parametric mixer architecture," Nature Photonics, vol. 5, no. 12, pp. 748-752, Dec. 2011.

[3] K. Cvecek, et al., "Phase-preserving amplitude regeneration for a WDM RZ-DPSK signal using a nonlinear amplifying loop mirror", Opt. Express 16, 1923-1928, (2008).

[4] K. Croussore et al., "Amplitude regeneration of RZ-DPSK signals based on four-wave mixing in fibre”, Electron. Lett., 43 (3), 177- 178, (2007).

[5] M. Matsumoto et al., "Performance improvement of DPSK signal transmission by a phase-preserving amplitude limiter", Opt. Express 15, 8094-8103 (2007)

[6] K. Bottrill, et al., "Investigation into the role of pump to signal power ratio in fwm-based phase preserving amplitude regeneration," in "CLEO: 2015," (Optical Society of America, 2015), p. SM2M.1.

[7] M. Matsumoto, "Phase noise generation in an amplitude limiter using saturation of a fiber-optic parametric amplifier," Opt. Lett. 33, 16381640 (2008).

[8] K. Kikuchi et al., "Compensation for pulse waveform distortion in ultra-long distance optical communication systems by using midway optical phase conjugator", IEEE Photonics Technol. Lett. 6, 104-105 (1994). 